\title{
AGRONEGOCIOS Y SUS POTENCIALIDADES PRODUCTIVAS RUMBO AL BICENTENARIO
}

\section{AGRIBUSINESS AND ITS PRODUCTIVE POTENTIAL TOWARDS THE BICENTENNIAL}

\author{
Uriel Rigoberto Quispe Quezada ${ }^{*}$ \\ uquispe@unah.edu.pe \\ ${ }^{1}$ Universidad Nacional Autónoma de Huanta, Ayacucho, Perú \\ *Correspondencia: Uriel Rigoberto Quispe Quezada. Email: uquispe@unah.edu.pe
}

Recibido: 09.06.21 | Aprobado: 16.07.21

\section{RESUMEN}

El aporte de los agronegocios de cara al bicentenario en el Perú, está siendo representada cada vez más con mayor prestancia en los mercados internacionales y la comunidad científica, por las bondades que ofrece a través de los cultivos con altísimo valor nutricional y alimentario, rescatada en algunos casos, por su apreciable valor genético de los recursos que se encuentran en los bancos de germoplasma, en cuyas especies es vasta y el número de accesiones y líneas cada vez cobra importancia, en los usos que se les da, que contribuye opciones para su industrialización y recibida por los consumidores que aprecian su riqueza nutricional y colma expectativas. El objetivo de la investigación aborda conocer las múltiples potencialidades de cultivos de exportación, promovidos a través de los agronegocios de cara al bicentenario en el país. En cuanto a la metodología aborda tomando en referencia niveles de análisis de cómo se ha venido comportando los cultivos bandera en los mercados internacionales apoyadas con la utilización y sistematización de fuentes variadas de información, en cuya incisión investigativa aborda con capacidad crítica. Las conclusiones que se presenta a lo largo de décadas, los cultivos de bandera están teniendo su participación, un lugar expectante en los ambientes comerciales, resaltando sus potencialidades al presentar riqueza nutraceútica, las cuales son utilizadas como fuente de alimento para el consumo humano y a la vez emplaza la participación de éstas en un mundo cada vez más competitivo.

Palabras clave: Agronegocios, potencialidades, cultivos, productivas.

\begin{abstract}
The contribution of agribusiness in the face of the bicentennial in Peru, is being represented more and more with greater presence in international markets and the scientific community, for the benefits it offers through crops with high nutritional and food value, rescued in In some cases, due to the appreciable genetic value of the resources found in germplasm banks, in whose species it is vast and the number of accessions and lines is becoming increasingly important, in the uses that are given to them, which contributes options for their industrialization and welcomed by consumers who appreciate its nutritional richness and meet expectations. The objective of the research addresses knowing the multiple potentialities of export crops, promoted through agribusiness in the face of the bicentennial in the country. Regarding the methodology, it addresses taking in reference levels of analysis of how flagship crops have been behaving in international markets supported by the use and systematization of various sources of information, in whose investigative incision it addresses with critical capacity. The conclusions that are presented over decades, the flag crops are having their participation, an expectant place in commercial environments, highlighting their potentialities by presenting nutraceutical richness, which are used as a source of food for human consumption and for instead it places their participation in an increasingly competitive world.
\end{abstract}

Keywords: Agribusiness, potential, crops, productive.

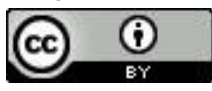




\section{INTRODUCCIÓN}

Los agronegocios en el Perú, incluye actividades productivas, industriales, exportadoras y comerciales (Miguel García et al., 2009). Cada sector se interrelaciona a través de productos que conforman la oferta agroexportable a lo largo de cadenas agroalimentarios (Miguel García et al., 2009). Agronegocios es un sistema que agrupa varias fases que van desde la producción del campo, transformación, almacenamiento, distribución y comercialización (Maiz et al., 2020). Para Castro et al., (2018), el sistema de los agronegocios peruano incluye desde las actividades productivas, industriales, exportadoras y comerciales. Estas se interrelacionan a través de productos que conforman la oferta agroexportable a lo largo de cadenas agroalimentarias (Castro et al., 2018)

Es innegable de la capacidad que posee el país, en poseer una gran variedad de cultivos en calidad de exportación, recursos genéticos de alto valor, biodiversidad de especies vegetales invaluables, cada uno de ellos desde su espacio, vienen contribuyendo al desarrollo de país, colocándolo un lugar cada vez más expectante en el mercado internacional. Recursos genéticos con incalculable valor, que muchos de los países ya desearían tener, poniendo al Perú en la cima de una riqueza tradicional, desde lo prehispánico y utilizadas para muchos fines de investigación, en la búsqueda constante de identificar potencialidades para usos comerciales. Utilizadas por la comunidad científica, tomando prestancia en las diversas investigaciones y en cuyos resultados viene generando aportes en solucionar problemáticas y contribuyendo a generar valor agregado.

La presencia en los países de desarrollo se ve con mayor prestancia cultivos altoandinos de gran riqueza tradicional y nutracéutica, actualmente bien valorada por los consumidores de estos países al tener como alimento base en su dieta alimenticia, por la gran problemática en su población de sobrepeso, exceso de ingesta de carbohidratos que ocasiona problemas cardiacos y entre otros., cuando en el Perú estos alimentos se ha venido consumiendo a lo largo de las décadas en su dieta familiar, desde lo prehispánico hasta la actualidad.

En la actualidad el país tiene la oportunidad de liderar, en particular, el sector agroexportador mundial, debiendo enfrentar el reto de potenciar su producción en cantidad y calidad, así como la generación de valor agregado (Castro et al., 2018). En general, la mayor parte de estudios buscan identificar la influencia de diferentes factores como la competitividad (Castellanos, 2012), la asociatividad (Cedeño, 2015) y la sostenibilidad (Junca \& Quispe, 2016), pero de manera individual, sin identificar sinergias o inconsistencias en las estrategias comerciales (Castro et al., 2018), y las oportunidades (Geldes \& Felzensztein, 2013).

La promoción de estos cultivos en los ambientes internacionales, propició mayores compras y precios competitivos de productos con alto valor nutritivo, en muchos de los casos consolidándose su presencia en los mercados con calidad de exportación, Las superficies de áreas cosechadas durante el periodo 2004 al 2018 crecieron en un 258\%, representando 57,441 ha y 205,600 ha respectivamente. (PROMPERU, 2019). 


\section{MÉTODO}

Esta investigación es considerada descriptiva bajo el enfoque cualitativo, tomando en consideración el uso y sistematización de fuentes variadas de información procedente de artículos científicos de investigación, revistas especializadas, libros, boletines informativos y medios electrónicos, las cuales son analizadas de manera crítica, abordando la problemática de los agronegocios frente al bicentenario.

\section{PERSPECTIVAS Y POTENCIALIDADES DE GRANOS ANDINOS}

El caso de la quinua, producto emblemático del país, que en los últimos años vienen consolidándose como uno de los alimentos con múltiples bondades nutricionales. Toma relevancia mundial después de la promoción que se le ha dado en el año 2017, declarándose año internacional de la quinua, donde los niveles de importación han sido significativos en relación al promedio normal de estos productos. Alcanzando precios muy competitivos, en cuya alza estuvo expectante logrando cubrir los costos productivos por parte de los agricultores. Posteriormente, a la falta de promocionar adecuadamente por parte del gobierno, y ejercer una política de conservar el recurso genético, hoy en día muchos de los países en el mundo vienen sembrando estos productos y logrando altos niveles de producción en comparación al Perú.

La existencia de pérdida de material genético le está costando al país, perdiendo niveles de posicionamiento en estos rubros, a la falta de una política de nivel de seguridad y proteccionismo nacional, que beneficiaría a muchas poblaciones altoandinas que ven como su fuente de recurso económico que al menos cubra sus expectativas y reditúe ciertos niveles de utilidad.

Citar, el caso de la quinua un grano con alto valor proteico, que aporta proteínas, ácidos grasos insaturados y minerales; kiwicha conocida también como amaranto, alimento de excelencia para los incas, debido a sus propiedades nutritivas y medicinales, contiene un alto valor en proteínas y minerales como calcio, fosforo, potasio, zinc y otros; Cañihua cultivo que prospera en el altiplano peruano resistiendo a las heladas y sequías (UnahALDIA, 2020a).

Destacando sus propiedades nutricionales un alto contenido proteico y un balance de aminoácidos como lisina, isoleucina y triptófano, posee además vitaminas E y complejo B; Sacha Inchi, planta oleaginosa de la Amazonía peruana, cuyas semillas contienen una de las más importantes fuentes de omega 3, 6 y 9 del mundo; Camu Camu pequeño fruto ovalado de superficie lisa, color rojo y centro ácido, contiene un alto índice de vitamina C; Lúcuma sus beneficios nutricionales destacan que aporta vitaminas y minerales; Aguaymanto poderoso antioxidante con altos contenidos de vitaminas A, B y C., (Noticias, 2017) y entre otros alimentos de importancia económica (UnahALDIA, 2020b).

Otros de los productos que tiene mayor prestancia en el mundo comercial es el amaranto, este cultivo antes de la presencia del cultivo de la quinua ha tenido su posicionamiento comercial en el mundo, e incluso por sus bondades nutricionales ha sido galardonada por organismos internacionales.

$\mathrm{Al}$ igual que estos también se traduce hoy con mayor prestancia el cultivo de la cañihua, utilizándose en una diversidad de usos, desde los ya conocidos y ampliamente 
difundidos en el mercado nacional los jugos de cañihua, quinua y amaranto, acompañados por una variada gastronomía, muy valorada por los consumidores locales y mercados internacionales (Pasco, 2016).

Otra de las especies es el cultivo de la maca, producido en las zonas altoandinas en las regiones de Junín, Pasco Huancavelica y algunas zonas altiplánicas, su potencialidad en encuentra en la obtención de harinas siendo el mercado principal Estados Unidos, algunos países de la región asiática el Japón, con el avance se ha logrado consolidar en la obtención de certificaciones orgánicas, que les ha permitido ingresar con valor agregado y de manera directa a los mercados de Estados Unidos y la Unión Europea, manteniendo esa característica de que en el país se producen alimentos de calidad, libres de insumos químicos y respetando el medio ambiente (Pasco, 2016). No solamente se queda ahí sino también en la obtención de diversos productos derivados caso licores, infusiones y entre otros.

Productos orgánicos toman mayor relevancia, según Suca, (2004), en especial se toma atención en la calidad, poseer precio de venta competitivos y lograr una buena diferenciación de los productos peruanos, son tres aspectos fundamentales para que las agroexportaciones peruanas tengan éxito en los mercados internacionales, como productos orgánicos de la unidad productiva.

\section{PRODUCTOS FRESCOS BANDERA DE CALIDAD EXPORTABLE}

Cultivos de representación nacional considerados a nivel de producción dentro del top ten en caso del espárrago, muchas de las agroexportadoras siembran a gran escala y posteriormente éstas vienen siendo procesadas en sus diversas presentaciones y combinadas con otros, poniendo a disposición en los mercados internacionales.

En varias décadas el Perú sigue siendo uno de los países con altos niveles de producción y venta manteniendo volúmenes de producción e importación de estos productos por parte de empresas en el exterior. Mas allá de los hábitos de consumo, éstas poseen un mercado potencial claramente definido y a lo largo de la franja costera aún se está en plenas campañas de producción, industrialización y exportación. Sumadas a éstos cultivos, la páprika que es uno de los ajíes de mayor salida y alta potencialidad que también en el país se viene promoviendo activamente en su producción e industrialización, e incluso muchas de las tierras agrícolas con instalaciones de cultivos permanentes el caso de la alfalfa, y otras especies en las regiones ganaderas han venido cambiando el giro de producción, tomando prestancia las instalaciones de estos cultivos que cada vez más demandan mayores producciones para su venta externa.

La alcachofa, sigue siendo un cultivo de mayor prestancia, a lo largo de muchos años, se han presentado muchas alternativas a fin de lograr altísimos niveles de producción, con la dotación de cultivares a nivel de precocidad, resistencia y capacidad de producción. Este también interviene en los procesos de industrialización que se da por diversas presentaciones, muy reconocido en la comunidad exterior y al ser cultivos estacionales le permite cubrir adecuadamente en los meses de estiaje a nivel internacional. En muchos de los sectores del Perú, se presenta este tipo de cultivares, no obstante, el grueso de la población del país aún no conoce sobre las potencialidades de este cultivo, 
ni más tener los hábitos de consumo que le harían tener mayores probabilidades de que su participación sea de manera sostenible.

Al igual que éstas, muchos de las especies vegetales están siendo cada vez más demandada, por los consumidores y viene sentando alternativas en su industrialización, como son las papas andinas, estos productos actualmente viene siendo insumos en el procesamiento de papas saladas, presentes en los ambientes gastronómicos, comerciales, la característica es que presentan diversas tonalidades y es producto de una variada nivel genético de estas especies, muchos de ellas agrupadas en los bancos de germoplasma, donde el nivel de acciones son debidamente conservadas y sembradas a fin de reponer su potencialidad genético.

La contribución hacia el bicentenario, se mira expectante, en continuar apostando por las especies naturales, caso de camu camu, cultivo de calidad de exportación con múltiples oportunidades en su industrialización, especies de vegetales que son consideradas medicinales en caso de guion, uña de gato, mashua, y otros.

\section{SUPERFRUTAS PERUANAS QUE CONQUISTAN MERCADOS}

La existe de frutas de calidad de exportación aún mantienen niveles expectantes de comercio, el caso del mango peruano, sembrando a lo largo de franja costera, hasta llegar a los climas cálidos para su producción y posterior exportación, muy demandado en los mercados asiáticos, quienes valoran por sus niveles de consistencia, sabor, aroma, cuerpo, y carnosidad,

El caso de la uva de mesa, las especies de redglobe, también es otro de los productos que cosechan muchas de las empresas de agroexportación en la franja costera, a través de una buena política de manejo controlado de plagas y enfermedades, obtención de certificaciones de sus instalaciones, campos de cultivo aseguran que la producción sea de altísima calidad, y posteriormente representa el insumo principal para su procesamiento e industrialización, guardando niveles de control permanente a nivel de certificaciones de sus instalaciones y campos de cultivo, que le hacen ser fuente de insumos para la obtención de diversos productos por las empresas,

A estos se suma otro de los productos vadera el caso de la lúcuma, fruto proveniente de la familia de las sapotáceas originario de los valles interandinos en el Perú, fruta muy utilizada en la elaboración de postres y helados, así como insumo principal para usos industriales en la obtención de deshidratados y entre otros.

\section{REDUCCIÓN DE BRECHAS ENTRE PRODUCTOS TRADICIONALES Y NO TRADICIONALES}

En el Perú, el sector agroexportador se ha constituido, en los últimos veinte años, en la segunda actividad económica con mayor rentabilidad después de la minería, multiplicándose casi ocho veces sus ganancias a nivel de exportaciones de productos no tradicionales (Castro et al., 2018). Hace varias décadas el Perú en inminentemente exportador de productos primarios, llegando hasta un nivel del $70 \%$ promedio, no obstante, estos altos porcentajes se han visto reduciendo por la presencia de mayor incremento en las exportaciones no tradicionales, PROMPERU, (2019). En el año 2000 llegaban a 95 mercados con un valor cercano a los US $\$ 400$ millones; en el 2019 se 
incrementaron a más de US $\$ 6,292$ millones cubriendo 145 mercados internacionales (Economía, 2019).

Este último en base a un crecimiento sostenido por la presencia e importancia de que el país viene apostando por la agroexportación, teniendo una vasta condición geográficas que le permiten poder cultivar tierras de cultivos de exportación y condiciones de infraestructura de riego, que asegure dotar del recurso hídrico indispensable para el desarrollo de las plantas y asegure las cosechas bajo los estándares de calidad y normas técnicas exigentes en los mercados internacionales. De los más de 5 millones de hectáreas que constituyen la superficie agrícola del Perú, alrededor de 100,000 corresponden a una agricultura moderna. Estas son responsables de los casi US\$ 3,333 millones de exportación no tradicional y parte de los US\$1,168 millones de exportación tradicional, excepto el café que se cultiva en las zonas medias altas de nuestra Amazonia (CONEXIONESAN, 2011).

A partir de los acuerdos comerciales en el 2000 y la expansión de la frontera agrícola con los grandes proyectos de irrigación en la costa, las exportaciones agrarias han venido creciendo exponencialmente (Gestión, 2018), posicionado al Perú entre los 20 principales proveedores de alimentos en el mundo de productos como la quinua, espárrago, café, mango, maca, aceituna, uva, palta, alcachofa, pimientos secos, frijol, mandarina, jengibre, habas, cebollas, arveja fresca y recientemente arándanos, cultivos que tienen además un enorme valor en términos de su aporte a la seguridad alimentaria mundial (Gestión, 2018).

No solamente la importancia de apostar por la agroexportación a lo largo de franja costera donde se encuentran la mayor proporción de áreas de cultivos potenciales, sino que además estas actividades se orientan en la sierra y en el oriente del Perú, cuya contribución si bien es cierto es limitada pero no menos importante a escala nacional, de ahí su importancia de orientar todos los esfuerzos necesarios en promocionar desde las instituciones públicos y privadas hacia la internacionalización, con una serie de mecanismos que beneficie a empresas de gran escala, mediana y pequeños emprendimientos, en su proceso de colocación de sus productos en el mundo.

\section{EXPECTATIVAS COMERCIALES EN EL MUNDO}

Las experiencias exitosas de empresas que han logrado ingresar a mercados internacionales, le ha abierto camino a nuevos escenarios y a la vez concretizar transacciones comerciales con clientes muy exigentes y competitivos, con la oferta de una cartera de portafolio diverso de productos de altísima calidad e innovadoras. Este esfuerzo ha requerido tiempo e inversión, con el apoyo en algunos casos de Instituciones nacionales en materia de comercio exterior, dotándolos en la capacidad organizativa y de presentación en las distintas ferias internacionales, ruedas de negocio, entrevistas con potenciales clientes, lográndose resultados positivos en factor de este sector.

Durante el año 2019, el Departamento de Agronegocios de PROMPERÚ estuvo presente en 14 ferias internacionales, 3 misiones comerciales itinerantes, 1 misión comercial local y 8 ruedas de negocios, generando más de US\$1,160 millones en oportunidades comerciales logradas a través de 10,290 citas comerciales gestionadas para 430 empresas peruanas expositoras. (PROMPERU, 2019) 
La apertura de nuevos mercados por parte del gobierno, constituye una oportunidad de negocios, en el sector empresarial, quienes pueden acogerse a una serie de beneficios que contemplan en dichos acuerdos. Es evidente sobre la participación del gobierno en ser un ente promotor de la industria nacional. El crecimiento de las exportaciones y diversificación de mercados de productos frescos se dio gracias a la apertura de mercados que pudieron ser concretadas durante periodo el periodo $2018 \mathrm{y}$ 2019.

En el 2018 se abrió el mercado de los cítricos hacia destinos como República Dominicana, Malasia y Japón; siendo la variedad beneficiada la mandarina satsuma para estos dos últimos destinos. Otros mercados que se lograron abrir gracias a mejoramientos de acceso fueron Malasia e Indonesia como sucedió para la uva fresca. Durante el año 2019, se concretaron accesos de mercado importantes como la palta hass fresca para Tailandia y Corea del Sur. Para el 2020 se proyecta que se pueda concretar nuevas aperturas mediante acuerdos sanitarios con países como Filipinas, Japón, India, Taiwán, China, Malasia, Colombia y Corea del Sur (PROMPERU, 2019)

\section{PANDEMIA Y SUS EFECTOS EN LOS AGRONEGOCIOS}

Uno de los escenarios que se presentó en la última década, según Pulcha-Ugarte et al., (2020) a finales del 2019 en Wuhan (China), se originó a una pandemia que alcanzaría una escala global, comparándose con otras tan letales que se ha presentado en la historia de la humanidad. Esta es una neumonía viral atípica y altamente contagiosa, covid-19, cuya causa se le atribuye a un coronavirus de transmisión zoonótica al que se denominó SARS-CoV-2 (Pulcha-Ugarte et al., 2020b) (Quispe Quezada, 2020). Esta crisis sanitaria trajo severas consecuencias en los sectores comerciales y los agronegocios en el Perú no estuvo exento de esta problemática, afectando actividades comerciales en algunos rubros.

Perú fue el primer país de la región en decretar una cuarentena general obligatoria, el 15 de marzo. El presidente Martín Vizcarra también ordenó el cierre de sus fronteras durante un lapso de 15 días (Mundo, 2020). Después de la declaración de emergencia nacional, el sector se vio afectada, por la paralización que duró varios meses, por la incidencia de la pandemia, tal como lo evidenciaría posteriormente reportes del ejecutivo a mediados de abril del 2020, las pérdidas estimadas ascendían hasta S/. 1611 millones de soles en el sector agropecuario, afectando a pequeños agricultores, quienes no suelen tener acceso al sistema bancario, ni son beneficiarios de programas sociales del Estado.

El sector agroexportador registró caídas en sus niveles de exportación por la presencia del coronavirus a nivel mundial, que obligó a que los países implementen cuarentenas obligatorias a su población como medidas de prevención para afrontar la diseminación del virus y consecuentemente el cierre del comercio internacional entre países, decisiones drásticas que pusieron en serias dificultades al Perú para colocar sus productos al mercado, en muchos de los casos productos que se encontraban en los lugares de destino, no pudiendo ingresar por las medidas de confinamiento aplicadas (Quispe Quezada, 2020).

El cierre de las fronteras internacionales impuestas en los diversos países en el mundo, presentaron restricciones comerciales en las empresas a la hora de realizar 
contratos con el flete de las embarcaciones de carga, así como la exigencia en cumplir con la calidad de cartera como es sabido que se da por medio de las liquidaciones de destino. La entrada a la etapa de confinamiento en tiempos distintos, ha generado un desfase entre el suministro y la demanda entre los países que cerraron las fronteras para frenar la propagación del virus ejemplos como: China ingresó en cuarentena el 23 de febrero, seguida de Corea del Sur, Estados Unidos a finales de marzo, la Unión Europea que agrupa a varios países entró a mediados de marzo y el Perú hizo lo propio el 15 de marzo. (UnahALDIA, 2020a)

En el segundo semestre del año 2020, aun reflejaba problemas teniendo en cuenta contextos habituales en la que el inicio de las campañas agrícola en la siembre de cultivos iniciaba los primeros días del mes de agosto, no obstante, las paralizaciones afectarían la cadena de comercialización y en cuyas respuestas hacia los agricultores llegaría tardíamente. Frente a este escenario posteriormente por disposiciones del ejecutivo ponía a disponibilidad el Programa de Garantía para el Financiamiento Agrario Empresarial (FAE-Agro) (AS. COM, 2020), que aseguraba un desembolso de aproximadamente $\mathrm{S} / 2,000$ millones de soles para el otorgamiento de garantías a pequeños agricultores.

Los impactos que se generó por la pandemia evidenciarían que los sectores de una agricultura a menor escala no fueron tan severos tanto en sus niveles de producción al igual que los precios. no obstante, en los sectores mayores afectaron por los bloqueos internacionales al cerrar sus principales puertos de destino, las cuales fueron aperturando a medida que esta pandemia estuvieron bajo control pero que, sin embargo, en forma irregular, esto consecuentemente afectaría el nivel de las agroexportaciones peruanas a los mercados internacionales.

Los principales mercados a las que se destina son a la China, Estados Unidos, la Unión Europea, quienes son considerados socios estratégicos del Perú y permiten dinamizar las exportaciones no tradicionales. Alimentos, que cada vez más son demandados en los mercados internacionales con buenos precios, aun durante la crisis de la pandemia del covid-19 (iQué!, 2020), que ha generado un cambio de actitud en los consumidores en preferir alimentos naturales y saludables (UnahALDIA, 2020a).

En base a las conclusiones de investigadores quienes realizaron un análisis exhaustivo manifiestan que la pandemia no ha tenido afectación significativa en el sector agroexportador, en donde La tendencia de crecimiento, a pesar de que por mes no ha tenido aumento sino disminución, a nivel de acumulado trimestral o cuatrimestralmente hablando ha representado un porcentaje de crecimiento del sector agroexportador de manera positiva, manteniendo a sus principales destinos y productos, $\mathrm{y}$ hasta aumentando las exportaciones del mismo (Velarde Flores, 2020), (Apolino, A; Llontop, M; Acuña, M; Paredes, 2020)

\section{AGRADECIMIENTOS}

A la Universidad Nacional Autónoma de Huanta, por su incansable motivación a sus docentes en promover investigación y análisis en el ambiente académico- científico. 


\section{CONCLUSIONES}

El Perú país maravilloso, que posee riquezas invaluables, cuyos productos agrícolas provenientes de épocas prehispánicas, adaptadas a las condiciones altoandinas como la quinua, cañihua y amaranto, en las últimas décadas toma mayor relevancia e importancia en los mercados internacionales por sus propiedades nutraceúticas, rico en proteínas y aminoácidos, que favorece a la salud humana y es considerada una alternativa frente al consumo excesivo de grasas saturadas por la población, hoy en día se encuentra en los principales cadenas de minoristas en varios países del mundo, gracias al esfuerzo de varios años de promoción y posicionamiento del sector público y privado.

Hace muchos años, el país viene consolidando con sus productos agroexportables de bandera, éstas aun hasta el presente, sigue manteniendo su condición de líderes de producción en los mercados nacionales e internacionales, cumpliendo con los estándares de calidad e inocuidad fitosanitaria, siendo representados por el espárrago, alcachofa, páprika, y entre otros.

Frutos de consistencia carnosa- drupas, bayas: mango, durazno, palta, uva; cítricos, calidad de exportación, cuyos productos conquistan exitosamente mercados occidentales, europeos, asiáticos, por su capacidad de presentar características de consistencia, cuerpo, sabor, color, acidez, muy reconocidos por los empresarios importadores, por presentar en el país áreas con ventajas comparativas frente a otros, que le hacen ser únicos y competitivos, aún mantienen con expectativa negocios comerciales en la región y en otros ámbitos geográficos.

Productos con propiedades medicinales y orgánicas, en las últimas décadas están siendo investigadas, cuyos resultados cada vez más, cumplen con expectativas y se generan actividades comerciales, siendo la maca, mashua, yacón, camu camu y entre otros, y la vez generan nuevos productos a nivel procesados de altísima calidad.

La generación de agronegocios en el país hace varias décadas está tomando mayor importancia, generando movimientos comerciales, dinamizando el sector empresarialproductivo, contribuyendo al desarrollo nacional, mejorando calidad de vida en la población.

La contribución en la economía nacional proveniente de la actividad comercial en los agronegocios, está encaminada con expectativa frente al bicentenario, dejando épocas difíciles que tradujeron recesión económica, vulnerabilidad en los mercados internacionales y entre otras problemáticas, aún queda por doblegar esfuerzos hacia la consolidación con miras estratégicas en el sector.

La pandemia, en el rubro de los agronegocios, si bien es cierto que los impactos generados no han trascendido en su real magnitud, si ha tenido efectos en colocar productos a mercados internacionales y una de las formas que ha transcendido para aminorar estos efectos fue la diversificación de mercados, gracias a los convenios suscritos y acuerdos comerciales del Perú con otros países, sumadas a la reactivación económica temprana de países como China, Corea del Sur e India. A nivel interno los impactos han sido sopesados por la fortaleza de los productores a menor escala, quienes con su esfuerzo y sacrificio salieron adelante, pese a las carencias y dificultades en lograr acceder a 
oportunidades provenientes del ejecutivo. No obstante, aún existe brechas por superar y alcanzar los resultados en años anteriores.

Frente al bicentenario, queda aún trabajar y dar prioridad a estos sectores, que, por sus condiciones geográficas en muchas zonas del país, son consideradas de alto riesgo dependientes de factores climatológicos, que limita su capacidad de producción y alcance de rendimientos, limitando la disponibilidad de alimentos y con ello generar ingresos económicos.

\section{REFERENCIA BIBLIOGRÁFICA}

Apolino, A; Llontop, M; Acuña, M; Paredes, N. (2020). Agronegocios Perspectivas de Mercado. Impacto del COVID-19 en las agroexportaciones peruanas. file:///C:/Users/HP/Documents/agronegocios/AGRONEGOCIOS.-Perspecti vas-de-mercado-UNMSM_.pdf

AS.COM. (2020). Coronavirus en Perú: resumen y casos del 3 de agosto. https://peru. as.com/peru/2020/08/03/actualidad/1596441585_095146.html

Castellanos, E. R. (2012). La geopolítica de los agronegocios: Competitividad del mercado agroalimentario en el nuevo orden mundial. Revista CIFE: Lecturas de Economía Social, 14(21), 155. https://doi.org/10.15332/s2248-4914.2012.0021.06

Castro, H. L., Goicochea, C. U., \& Flores, M. F. (2018). El Sistema De Agronegocios En El Perú: De La Agricultura Familiar Al Negocio Agroalimentario. Revista Mexicana de Agronegocios, 43(2018), 1-16. https://www.redalyc.org/jatsRepo/141/14158 242001/14158242001.pdf

Cedeño, M. S. del M. R. (2015). De la agricultura arcaica al agronegocio y los modelos asociativos. Su impacto social. Journal of Agriculture and Environmental Sciences, 4(2), 137-145. https://doi.org/10.15640/jaes.v4n2a16

CONEXIONESAN. (2011). Potencialidades en agronegocios. https://www.esan.edu.pe /conexion/actualidad/2011/05/02/potencialidades-en-agronegocios/

Economía, A. (2019). Sector agroexportador del Perú proyecta incremento de inversiones privadas. https://www.americaeconomia.com/negocios-industrias/sector-agroex portador-del-peru-proyecta-incremento-de-inversiones-privadas

Geldes, C., \& Felzensztein, C. (2013). Marketing innovations in the agribusiness sector. Academia Revista Latinoamericana de Administración, 26(1), 108-138. https://doi.org/10.1108/arla-05-2013-0042

Gestión. (2018). Ley de Promoción Agraria y su impacto económico y social. https://gestion.pe/blog/innovacion-sinergias-y-crecimiento/2018/05/ley-de-pro mocion-agraria-y-su-impacto-economico-y-social.html/?ref=gesr

Junca, B., \& Quispe, A. (2016). Sostenibilidad de modos ancestrales de producción agrícola en el Perú: ¿conservar o sustituir? Mundo agrario, 17(35), 00-00. https://www.redalyc.org/pdf/845/84547331013.pdf

Maiz, F. D. E., Del, D. E. J., \& Cosecha, C. D. E. L. O. S. E. U. P. D. E. (2020). Perspectivas de Mercado. 202, 1-14. https://viceacademico.unmsm.edu.pe/wp- 
content/uploads/2020/10/AGRONEGOCIOS.-Perspectivas-de-mercado-UNM SM_.pdf

Miguel García, Riveros, H., Pavez, I., Rodríguez, D., Lam, F., Arias, J., \& Herrera, D. (2009). Cadenas agroalimentarias: un instrumento para fortalecer la institucionalidad del sector agrícola y rural. Comuniica, 26-38. https://goo.gl/plUdkL

Mundo, B. N. (2020). Coronavirus | Cómo hace frente al covid-19 cada país de América Latina.

Noticias, A. agencia peruana de. (2017). Día Mundial de la Alimentación: estos son los superalimentos peruanos. https://andina.pe/agencia/noticia-dia-mundial-dealimentacion-estos-son-super-alimentos-peruanos-728806.aspx

Pasco, L. (2016). Productores de maca obtienen certificación orgánica con apoyo del Mincetur. http://www.pascolibre.com/2016/11/productores-de-maca-obtienen. html

PROMPERU. (2019). Desenvolvimiento del comercio exterior agroexportador. https://www.siicex.gob.pe/siicex/resources/sectoresproductivos/Desenvolvimie nto Agroexportador 2019.pdf

Pulcha-Ugarte, R., Pizarro-Lau, M., Gastelo-Acosta, R., \& Maguiña-Vargas, C. (2020a). ¿Qué lecciones nos dejará el covid-19?: Historia de los nuevos coronavirus. Revista de la Sociedad Peruana de Medicina Interna, 33(2), 68-76. https://doi.org/10.36393/spmi.v33i2.523

Pulcha-Ugarte, R., Pizarro-Lau, M., Gastelo-Acosta, R., \& Maguiña-Vargas, C. (2020b). ¿Qué lecciones nos dejará el covid-19?: Historia de los nuevos coronavirus. Revista de la Sociedad Peruana de Medicina Interna, 33(2), 68-76. https://doi.org/ $10.36393 /$ spmi.v33i2.523

Qué! (2020). CONSEJOS ECONÓMICOS PARA SUPERAR LA CRISIS DE LA PANDEMIA DEL COVID-19. https://www.que.es/2020/09/10/consejoseconomicos-superar-crisis-covid-19/

Quispe Quezada, U. R. (2020). Pandemia COVID-19: Impacto en las Agroexportaciones Peruanas. En U. N. A. de Huanta (Ed.), Cuarentena Multidisciplinar (1.a ed., Vol. 1, Número 9, pp. 10-37). file:// C:/Users/HP/Downloads/7-Manuscrito de libro24-1-10-20200825 (3).pdf

Suca Apaza, F. (2004). Agronegocios. Boletin de la Maestria en Agronegocios, 3. UnahALDIA. (2020a). Sector agroexportador: desafíos frente al covid-19. https://www.aldia.unah.edu.pe/sector-agroexportador-desafios-frente-al-covid$19 /$

UnahALDIA. (2020b). Superfoods: Expectativa comercial postcovid-19. En Periodico digital unahALDIA. https://www.aldia.unah.edu.pe/superfoods-expectativacomercial-postcovid-19/ 
Velarde Flores, R. A. (2020). Agronegocios perspectivas de mercado. Journal of Chemical Information and Modeling, 1, 120. https://viceacademico.unmsm.edu.pe/wpcontent/uploads/2020/11/Agronegocios.-Perspectivas-de-mercado-UN-MSM2020.pdf\#page $=39$

\section{CITAR COMO:}

Quispe Quezada, U. R. (2021). Agronegocios y sus potencialidades productivas rumbo al bicentenario. Puriq, 3(3), 377-388. https://doi.org/10.37073/puriq.3.3.201 